\title{
Generalised dynamical systems soill deformation model
}

Paul G. Joseph PhD, PE, MASCE

President, Engineering Solutions, Boxboro, MA, USA

\begin{abstract}
When sheared past failure, particles in a soil element move into the steady-state structure at random shear strains, behaving as a simple friction-based dynamical system that closely models test data for a wide range of soils, stress paths and drainage conditions. This paper reviews this dynamical systems approach and generalises it as follows: for any stress path, particles move at random shear strains into the final structure corresponding to the applied stresses; that is, the same friction-based dynamical system-based model of shear to failure applies to any stress path, not just to those that lead to failure. The model being general, it can be specialised for the particular case of one-dimensional consolidation - that is, shear along the $K_{0}$ line. Such a specialisation reveals the commonly observed linear relationship between $e$ and $\log \bar{\sigma}_{v}$ for one-dimensional consolidation and hence supports the generalisation.
\end{abstract}

\section{Notation}

$C_{\mathrm{c}} \quad$ compression index

e void ratio

$e_{0} \quad$ void ratio at start of test

$J_{e} \quad$ void ratio due to load-bearing particles not being in the final structure, per unit stress

$J_{q}, J_{p} \quad$ initial nondimensional interparticle contact area of the load-bearing particles that are not in the final structure, per unit area of the sample, corresponding to $q$ and $\bar{p}$, respectively

$K$ ratio of lateral to vertical stress for one-dimensional consolidation

$K_{0} \quad$ ratio of lateral to vertical stress for normally consolidated one-dimensional consolidation

$\bar{P}_{c} \quad$ original consolidation stress

$\bar{p} \quad$ effective normal/confining stress, defined as $\left(\bar{\sigma}_{1}+\bar{\sigma}_{2}+\bar{\sigma}_{3}\right) / 3(\mathrm{kPa})$

$q \quad$ shear stress, defined as $1 / 3\left[\left(\bar{\sigma}_{1}-\bar{\sigma}_{2}\right)^{2}+\left(\bar{\sigma}_{2}-\bar{\sigma}_{3}\right)^{2}+\left(\bar{\sigma}_{3}-\bar{\sigma}_{1}\right)^{2}\right]^{1 / 2}$

$R \quad$ strain rate $(1 / \mathrm{s})$

$S_{\mathrm{u}} \quad$ undrained shear stress

$t \quad$ time since the start of the test

$u \quad$ pore pressure

$\alpha_{q}, \alpha_{p}, \alpha_{e}$ secant friction angles (degrees) corresponding to $q$, $\bar{p}$ and $e$, respectively

$\gamma \quad$ shear strain, defined as $1 / 3\left[\left(\bar{\varepsilon}_{1}-\bar{\varepsilon}_{2}\right)^{2}+\right.$ $\left.\left(\bar{\varepsilon}_{2}-\bar{\varepsilon}_{3}\right)^{2}+\left(\bar{\varepsilon}_{3}-\bar{\varepsilon}_{1}\right)^{2}\right]^{1 / 2}(\%)$

$\varepsilon_{1}, \varepsilon_{2}, \varepsilon_{3} \quad$ principal effective strains (\%)

$\kappa_{q}, \kappa_{p}, \kappa_{S_{u}}$ nondimensional interparticle contact area of the particles that are not in the flow structure, corresponding to $q, \bar{p}$ and $S_{\mathrm{u}}$, respectively

$\lambda_{q}, \lambda_{p} \quad$ rate of movement of particles into the final structure, corresponding to $q$ and $\bar{p}$, respectively, per unit of strain

$\bar{\sigma}_{1}, \bar{\sigma}_{2}, \bar{\sigma}_{3} \quad$ principal effective stresses

\section{Introduction}

The steady-state condition is a final condition that soils ultimately reach when sheared past failure. The steady-state condition occurs in a particulate mass for any loading and drainage condition where the shear stress breaks down the original structure and puts it into a new 'flow' structure. It is the result of a continuous process that starts with the first small increase of shear stress and ends when the soil reaches a steady state of deformation, with all trace of the original structure lost and with a statistically constant structure. The steady-state condition applies to both drained and undrained shear.

At the steady state, particles continuously deform at constant shear stress, normal effective stress, volume and velocity. Poulos (1981) first formally defined the steady-state condition, and this paper relies on his strict definition.

At the steady state, all initial soil structure is lost; the steady-state condition is a function of the steady-state void ratio and, to a very small extent, of strain rate. In the case of an undrained test sheared to the steady state without any nonuniformities (shear bands) in the test specimen, the final (that is, steady-state) void ratio is the same as the initial void ratio; consequently, all undrained tests conducted with the same initial void ratio converge to the same steady-state condition regardless of overconsolidation ratio (OCR). This convergence (attraction) to the same single final condition, expressible as a point in the phase space (defined by the axes of void-ratio $e$, shear stress $q$ and confining stress $\bar{p}$ ) of the soil deformation system, is a characteristic of a dynamical system. Dynamical systems theory calls this final condition a 'point attractor'. All the steady-state point attractors together define the steady-state line.

The steady-state concept finds extensive use in analysing conventional geotechnical engineering problems as well as earthquake engineering, large-strain and soil dynamics problems (see e.g. Castro et al., 1985, 1992; Finno et al., 1996; Fourie and 
Tshabalala, 2005; Lade and Yamamuro, 2011; Okada et al., 2005; Poulos et al., 1985; Riemer and Seed, 1997; Wang and Sassa, 2002; Yamamuro and Covert, 2001; Yamamuro and Lade, 1998).

Desai (2001) and Desai et al. (2005) have proposed the 'disturbed state' concept, which includes both the critical state and the asymptotic final (steady) state. Others have also researched microstructural models and used them in discrete-element and finiteelement approaches - for examples, see O'Sullivan (2011), Yan and Dong (2011), and Zhou and Randolph (2007). The generalised soil shear microstructure-based deformation model presented in this paper complements their work.

Per the strict definition of the steady state, there are two differences between the steady-state and the critical-state conditions. These are that $(a)$ the steady state explicitly requires that deformations occur at a constant deformation velocity (which can be any velocity that does not cause inertial effects) and not only, as in the case of the critical state, at the near-zero (that is, pseudo-static) velocity; and (b) at the steady state the soil grains position themselves in the steadystate flow structure that remains statistically constant with continued shear strain - no such restriction applies to the critical state.

Strain-rate effects during shear, though small, are empirically evidenced - for an excellent summary see Díaz-Rodríguez et al. (2007). For the steady-state condition, this dependence on strain rate is very small; in practice, effectively zero. LaGatta (1970, 1971) suggested a heuristic of a $10 \%$ change in the steady-state strength for every three orders of magnitude change in strain rate. Nonetheless, theoretical completeness requires the additional specification of strain rate for the steady-state condition over and above that for the critical-state condition.

Soil particles move and reorient continuously during shearing and ultimately reach a steady-state flow structure that is statistically constant. Even in the steady-state flow structure, for elongated particles, there can be small, continual, random up/down and left/right movement, while for bulky grains, there can be continual, random grain rotations. However, the steady-state flow structure is statistically constant in that the movements of these individual particles result in negligible changes in the shear stress, normal effective stress, volume and velocity observed with continued straining at the steady state (Poulos, 1981 and personal communication to the author, 2010). One example of a steady-state condition is the well-known residual condition resulting from large strains of clays.

These differences between the steady state and the critical state are not a matter of semantics but have deep implications. They allow for the application to the steady-state concept of soil shear the mathematical framework commonly known as 'dynamical systems'. Dynamical systems theory, however, cannot be applied to the critical state, which does not meet the requirements of the steady state.

Dynamical systems are ubiquitous in nature - various other systems in nature, like soils, dynamically evolve from various initial conditions to the same final steady-state condition. Because of the static nature of soil's steady-state point attractor, the soil-shear dynamical system is a rather 'simple' dynamical system, as compared to dynamical systems that demonstrate attractors that may be 'complex' or 'chaotic'. (The terms 'complex' and 'chaotic' have strict technical meanings in dynamical systems theory (see e.g. Strogatz, 1994).)

Dynamical systems theory is a very active research area in the general sciences - scientists and mathematicians extensively study such systems and current undergraduate programs in mathematics and the sciences offer courses in dynamical systems theory. A good undergraduate-level textbook on the subject that exhaustively covers the dynamical systems concepts used in this paper is by Strogatz (1994). In short, though the dynamical systems soil deformation model does not appear to conform to standard elastoplastic soil mechanics, it nonetheless rests squarely on a scientific framework that is well known in the sciences.

\section{Dynamical systems applied to the shear of soils to failure}

This section briefly reviews the dynamical systems theory of soil shear and sets the stage for the generalisation of the theory to any stress path, including that for one-dimensional consolidation.

Joseph $(2009,2010)$ proposed a phenomenological model for soil shear based on two hypotheses. These are that $(a)$ the rates of change of shear stress, effective normal stress and void ratio are proportional to their applied values, with the initial constants decaying exponentially with shear strain to reach ultimately a value of 0 at the steady-state condition, and (b) shear stress works to destroy soil structure, while confining stress works to preserve it.

Subsequently, Joseph (2012) provided the underlying physical basis for this phenomenological model. This physical basis derived from two scientific hypotheses: $(a)$ simple friction acting at interparticle contact areas of the load-carrying particles governs soil shear; $(b)$ particles move into the steady-state flow structure at random shear strains - that is, as a Poisson process.

Probability theory describes a Poisson process as a stochastic process that assumes that the interval between each pair of consecutive events is independent of any other event interval. The process gets its name from the French mathematician SiméonDenis Poisson. Many phenomena can be accurately modelled by Poisson processes; well-known examples are radioactive decay (Cannizzaro et al., 1978), telephone calls (Willkomm et al., 2009) and requests for a particular document on a web server (Arlitt and Williamson, 1997).

From the hypothesis that particles move at random shear strains to the steady-state flow structure, a Poisson process underlies shear deformation. In other words, the case for soil deformation is analogous to, for example, radioactive decay, except that instead of radioactive particles leaving the nucleus at random times, for 
soils, particles move to their final state at random shear strains from out of the population of load-bearing particles not in the final state. This means that the number of particles that remain not in their final state decays exponentially: for radioactivity, the number of radioactive particles in the nucleus decays exponentially with time, while for soils, the number of load-bearing particles not at the steady state decays exponentially with shear strain. For the case of soils, the appendix in the paper by Joseph (2012) has the detailed derivation of this exponential decay.

Joseph (2013a) shows why as the number of load-bearing particles in the shear zone that are not in the steady-state decays exponentially, so too does their interparticle contact area. The reason is the loadcarrying particles not in the final state have some distribution of interparticle areas, and since these particles move to the final state at random during shear, the decrease in interparticle contact area of particles not at the final state comes randomly from within this distribution. This, in turn, means that the expected value of the per cent number of particles for any given interparticle contact area of this distribution is always the same. Because of this, decrease in total interparticle contact area across the distribution for each strain increment varies identically as the number of particles moving to the steady-state structure - that is, exponentially.

Furthermore, on the hypothesis of simple friction operating on the interparticle contact areas, the resulting model is

\section{1a. $\mathrm{d} q / \mathrm{d} \gamma=J_{q}\left[\bar{p} \tan \alpha_{q} \exp \left(-\lambda_{p} \gamma\right)-q \exp \left(-\lambda_{q} \gamma\right)\right]$}

1b. $\quad \mathrm{d} \bar{p} / \mathrm{d} \gamma=J_{p}\left[\bar{p} \tan \alpha_{p} \exp \left(-\lambda_{p} \gamma\right)-q \exp \left(-\lambda_{q} \gamma\right)\right]$

1c. $-\mathrm{de} / \mathrm{d} \gamma=J_{e}\left[\bar{p} \tan \alpha_{e} \exp \left(-\lambda_{q} \gamma\right)-q \exp \left(-\lambda_{q} \gamma\right)\right]$

where $e$ is the void ratio; $q$, shear stress; $\bar{p}$, effective normal/ confining stress; $J_{q}, J_{p}$, initial nondimensional interparticle contact area of the load-bearing particles that are not in the final structure, per unit area of the sample, corresponding to $q$ and $\bar{p}$, respectively; $J_{e}$, void ratio due to load-bearing particles not being in the final structure, per unit stress; $\alpha_{q}, \alpha_{p}, \alpha_{e}$, secant friction angles corresponding to $q, \bar{p}$, and $e$, respectively; $\gamma$, shear strain; and $\lambda_{q}, \lambda_{p}$, rate of movement of particles into the final structure, corresponding to $q$ and $\bar{p}$, respectively, per unit of strain. $J_{q}, J_{p}$, and $J_{e}$ are expected to be close to each other and, likewise, $\alpha_{q}, \alpha_{p}, \alpha_{e}$ and $\lambda_{q}, \lambda_{p}$; that is, the system is effectively defined by three parameters, with actual values varying slightly about these parameters.

Equations 1a-1c are implicitly time dependent - to express it explicitly in terms of time $t$, use the relationship $\mathrm{d} q / \mathrm{d} \gamma=$ $\mathrm{d} q / \mathrm{d} t * \mathrm{~d} t / \mathrm{d} y=\mathrm{d} q / \mathrm{d} t * 1 / R$, where $R$ is the instantaneous strain rate. The parameters of Equations $1 \mathrm{a}-1 \mathrm{c}$ are themselves strainrate independent, with the exception of the secant friction angles $\alpha_{q}, \alpha_{p}, \alpha_{e}-$ to the extent that these friction angles depend on the material's underlying coefficients of static and dynamic friction, Equations $1 \mathrm{a}-1 \mathrm{c}$ depend on strain rate. In the context of a constant strain-rate shear test, replacing shear strain $\gamma$ by $R t$ and the change in shear strain $\mathrm{d} \gamma$ by $R \mathrm{~d} t$, where $t$ is the time since the start of the test and $R$ is the constant strain rate of the test, both strain rate and time become explicit.

Note that the right-hand side of Equations $1 \mathrm{a}-1 \mathrm{c}$ is in terms of strain, not of strain increment. In general, an element of ground that has been perfectly sampled or perfectly tested in situ is at an unknown initial strain. Hence, strain increment is the preferred term with which to formulate constitutive models because strain increments are knowable, being a measurable change in element geometry. Alternate 'state variables' that can be measured, void ratio for example, also suffice for this purpose - systematically replace the shear strain in the right-hand side with the void ratio to bring Equations 1a-1c into this form. At the steady state, Equations $1 \mathrm{a}$ and $1 \mathrm{~b}$ will continue to evaluate to zero because the interparticle contact areas of particles in the shear zone that are not at the steady state are all zero, and Equation 1c will also be zero because at the steady state $J_{e}$ is zero. A demonstration of this change to using void ratio occurs later in this paper when Equations $1 \mathrm{a}-1 \mathrm{c}$ are specialised for the case of one-dimensional consolidation.

As in Joseph $(2009,2010)$, Equations 1a-1c continue to describe a dynamical system with a fixed, single-point attractor (the steady-state condition) defined by the steady-state void ratio (and, negligibly, by the strain rate). Given that the attractor is a fixed point, behaviour is not chaotic; stress paths for various different initial conditions converge to this steady-state point attractor as described by Poulos (1981). A measure of convergence used in dynamical systems theory is the Lyapunov exponent. For soils, the Lyapunov exponents are negative. This is expected because negative Lyapunov exponents are characteristic of dissipative that is, nonconservative - systems, such as is the case for soil deformation.

The global phase space of the soil deformation dynamical system has three dimensions, $q, \bar{p}$ and $e$. The global attractor is the steadystate line in positive $q, \bar{p}$ and $e$ space ( $e$ will not physically be able to take on negative values despite the form of Equation 1c). All the steady-state attractor points make up the steady-state line. Poulos et al. (1985) suggest as a heuristic for clayey materials that the projection of the steady-state line onto the $e, \bar{p}$ plane is parallel to the materials' normal consolidation line. The steady-state line will be slightly different to the extent that each steady-state point that constitutes it depends slightly on strain rate; that is, the steady-state line is a two-dimensional surface if strain-rate effects are included, but is a one-dimensional line otherwise.

It is possible to express Equations 1a-1c as a single homogeneous, second-order, linear equation for $q, \bar{p}$ or $e$ with nonlinear, nonautonomous coefficients. However, these equations have no closed-form solution. These equations show (Joseph, 2012) that $q$, $\bar{p}$ and $e$ behave like a mass-damper-spring system with both damper 
and spring nonlinear. Damping and spring stiffness both decay as they approach the steady state where damping reaches a constant value while spring stiffness becomes zero.

Joseph (2013b) shows that the model has a closed-form solution when $\lambda_{q}$ and $\lambda_{p}$ are restricted to be equal. Numerical solutions that make this assumption provide reasonable fits that typically differ from the measured values by less than about $25 \%$. However, the analytical solution that makes the same assumption provides poor fits to the measured data because it strongly depends on hard-tomeasure boundary conditions, particularly those at the start of plastic deformation. Consequently, the analytical solution usually provides only an approximate match to the data.

The dynamical systems model described by Equations 1a-1c does not describe elastic strains. Nor does it apply to cemented soils, meta-stable soils or soils with significant particle breakage on shearing. It applies, however, to any stress path, including, as shown by Joseph $(2009,2012)$, extension stress paths; that is, in theory, the model can also account for dynamically reversed loadings.

Joseph (2012) applied Equations 1a-1c to 130 shear tests from various research programs on uncemented clays, silts and sands under a variety of test conditions in triaxial, plane-strain and truetriaxial tests, and showed that the model provides close fits to large strains for the data together with an orderly variation of the model parameters. Standard fourth-order Runge-Kutta numerical integration calibrated the model to measured data to obtain the model parameters $J, \lambda$ and $\alpha$ corresponding to $q$ and $\bar{p}$ (and for the drained case, $e$ ) for a given test. For an undrained test, obtain pore pressure $u$ from the calculated values of $q$ and $\bar{p}$, and from the initial consolidation stress. Figure 1 plots against OCR the parameter values averaged regardless of strain rate for the Joseph (2012) model calibrated with compression triaxial tests run at different strain rates by Sheahan (1991). (Note: for clarity, Figure 1 plots the small values of $\alpha_{q}$ and $\alpha_{p}$ against the axis on the right.)

The figure shows a smooth variation of the parameters with OCR with high correlation coefficients, indicating a minimal dependence on strain rate. As expected from the underlying stochastic model, the regression equations for $J_{q}, \lambda_{q}$ and $\alpha_{q}$ are very close to those for $J_{p}, \lambda_{p}$ and $\alpha_{p}$, respectively. In fact, Joseph (2012) used the average of the pairs of parameter values, that is, just three parameters $J, \lambda$ and $\alpha$, to replace $J_{q}, \lambda_{q}$ and $\alpha_{q}$ and $J_{p}, \lambda_{p}$ and $\alpha_{p}$ in Equations $1 \mathrm{a}-1 \mathrm{c}$ to predict new shear test data.

A key feature of dynamical systems models is that, in theory, a small set of accurately measured data from the initial stages of a system's evolution suffices for the model to generate accurately complete system behaviour, all the way to the steady state. In practice, however, due to the effect of measurement inaccuracies, all available measurements help determine model parameters more precisely. As shown in detail by Joseph (2012), this feature of dynamical systems to be able to work with a very small but accurate data set allows the model to predict shear behaviour post formation of shear bands. Figures 2, 3 and 4 show typical fits of the model to data from Sheahan (1991), Arulmoli et al. (1992) and Shapiro (2000) for drained compression and extension tests on clay, sand and silt. Note the large strains to which accurate predictions from the model are possible.

Furthermore, for a given OCR, stress path and strain, all the relevant parameter values of the model are the same (see Figure 1); that is, for a given OCR and stress path, regardless of the initial stresses, the same frictional parameters govern shear-strain behaviour. From this, Joseph (2013b) showed consequently, as for the case of a block sliding in simple friction, that shear and normal stresses acting on the soil must normalise with respect to the initial normal stresses. In other words, the model provides a physical basis for why the entire stress-strain curve for undrained shear normalises for a given OCR and why, as a consequence, $S_{\mathrm{u}} / \bar{P}_{\mathrm{c}}$, the ratio of the undrained shear strength $S_{\mathrm{u}}$ to the original consolidation stress $\bar{P}_{\mathrm{c}}$, is approximately a constant for a given OCR.

Additionally, the model explains why a linear relationship exists between $\ln (\mathrm{OCR})$ and $\ln \left(S_{\mathrm{u}} / \overline{P_{\mathrm{c}}}\right)$ as follows. A measure of the nondimensional interparticle contact area of the particles not in the flow structure at any given strain is the summation of the coefficients of Equations 1a-1c integrated between the limits $\gamma=\infty$ (where, in the steady-state structure, this measure is zero) and any given strain $\gamma=\gamma_{i}$ as

$2 a$.

$$
\begin{aligned}
\kappa_{q} & =\int_{\infty}^{\gamma_{i}} J_{q} \exp \left(-\lambda_{q} \gamma\right)+\int_{\infty}^{\gamma_{i}} J_{q} \tan \alpha_{q} \exp \left(-\lambda_{p} \gamma\right) \\
& =\frac{J_{q}}{\lambda_{q}} \exp \left(-\lambda_{q} \gamma\right)+\frac{J_{q} \tan \alpha_{q}}{\lambda_{p}} \exp \left(-\lambda_{p} \gamma\right)
\end{aligned}
$$

$2 \mathrm{~b}$.

$$
\begin{aligned}
\kappa_{p} & =\int_{\infty}^{\gamma_{i}} J_{p} \exp \left(-\lambda_{q} \gamma\right)+\int_{\infty}^{\gamma_{i}} J_{p} \tan \alpha_{p} \exp \left(-\lambda_{p} \gamma\right) \\
& =\frac{J_{q}}{\lambda_{q}} \exp \left(-\lambda_{q} \gamma\right)+\frac{J_{p} \tan \alpha_{p}}{\lambda_{p}} \exp \left(-\lambda_{p} \gamma\right)
\end{aligned}
$$

where $\kappa_{q}$ and $\kappa_{p}$ are the nondimensional interparticle contact areas corresponding to $q$ and $\bar{p}$, respectively, of particles that are not in the flow structure at shear strain $\gamma_{i}$.

Equation $2 \mathrm{a}$ is the nondimensional interparticle contact area of particles not in the flow structure that corresponds to the shear stress observed at shear strain $\gamma_{i}$. Hence at the strain corresponding to the peak shear strength $S_{\mathrm{u}}$, Equation 2a gives $\kappa_{S_{\mathrm{u}}}$, the nondimensional interparticle contact area of particles not in the flow structure at the strain corresponding to the peak shear strength $S_{\mathrm{u}}$. Joseph (2012) showed that the relationships between $\ln \left(\kappa_{S_{\mathrm{u}}}\right)$ and $\ln (\mathrm{OCR})$ and $\ln \left(S_{\mathrm{u}} / \bar{P}_{\mathrm{c}}\right)$ are linear and that, consequently, a similar linear relationship exists between $\ln (\mathrm{OCR})$ and $\ln \left(S_{\mathrm{u}} / \bar{P}_{\mathrm{c}}\right)$; that is, the model provides an independent, alternate, quantitative and 


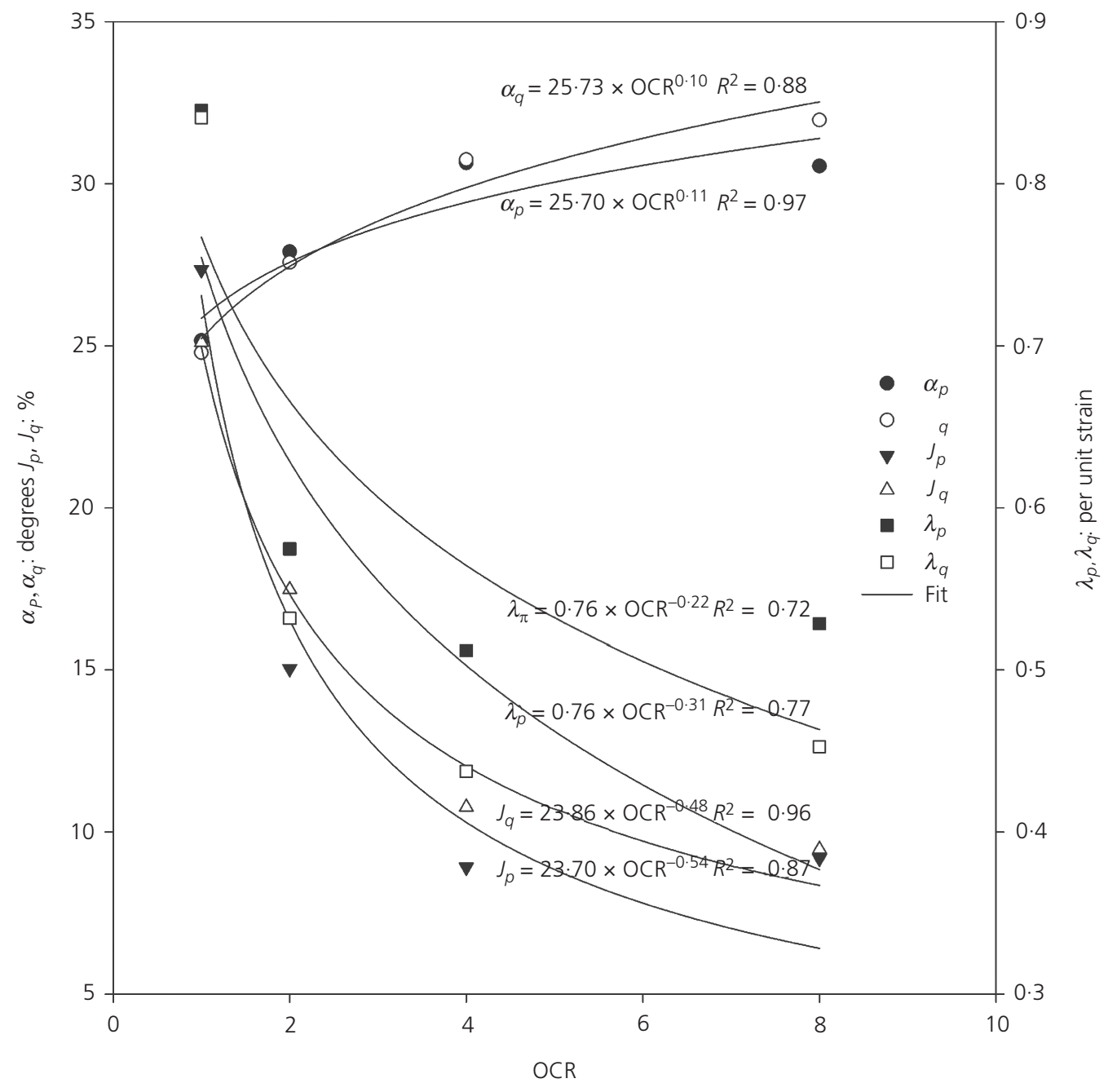

Figure 1. Model parameter values for Boston blue clay calibrated to Sheahan (1991) compression tests

structural basis for the empirical observation that $\ln \left(S_{\mathrm{u}} / \bar{P}_{\mathrm{c}}\right)$ varies logarithmically with OCR.

The close fits to the test data over a wide range of soils, test conditions and strains, together with the smooth variation of the curves of the model's parameters and with its successful predictions demonstrated by Joseph (2012), validate the two hypotheses that drive the model. The Joseph (2012) model is a physical model, not a phenomenological model. It is not 'curve fitting' because, unlike curve fitting, the parameters of the model have both direct physical meaning and scientific basis. The model's key significance is that it points to fundamental considerations underlying and driving soil shear. Its key advantage is the close fit to measured data, which, in turn, will result in better modelling of field problems.

Joseph and Graham-Eagle (2013) studied strain-rate effects on soil shear. They showed that plastic deformation begins at very small strains and that the early linearity of stress-strain curves during plastic deformation is due to the linear behaviour at very small strains of the nonlinear equations describing this plastic deformation. The soil particle's static coefficient of friction dominates at very small strains, after which the friction coefficient reduces to its dynamic value. Variations with strain rate in the stress-strain and void ratio-strain curves are small, due to the correspondingly small dependence of the soil particle's material coefficient's of friction on strain rate.

The model's significance is that it points to fundamental microstructural considerations that underlie soil shear. The model directly complements work done in areas that more immediately investigate micromechanical soil behaviour such as, for example, discrete-element methods (see e.g. O'Sullivan, 2011). Hence, the finding using discrete-element methods that only a minority of soil particles form a load-bearing skeleton that carries the bulk of 
Geotechnical Research

Volume 1 Issue 1
Generalised dynamical systems soil deformation model

Joseph

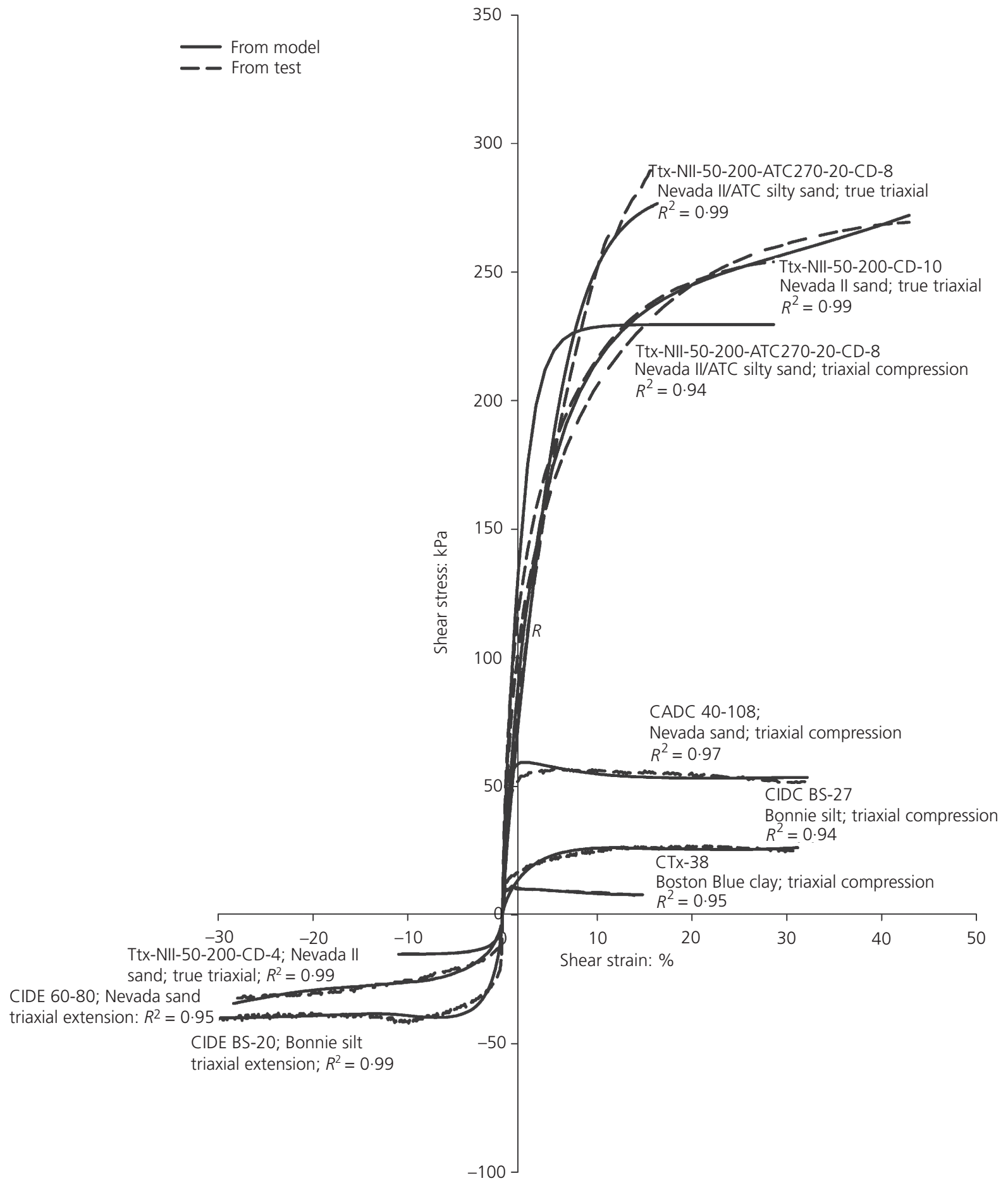

Figure 2. Measured plotted against calculated shear stress plotted against strain curves for nine sample shear tests (adapted from Joseph, 2012) 
Ttv-NII-50-200-CD-4; Nevada II sand true triaxial; $R^{2}=0.87$
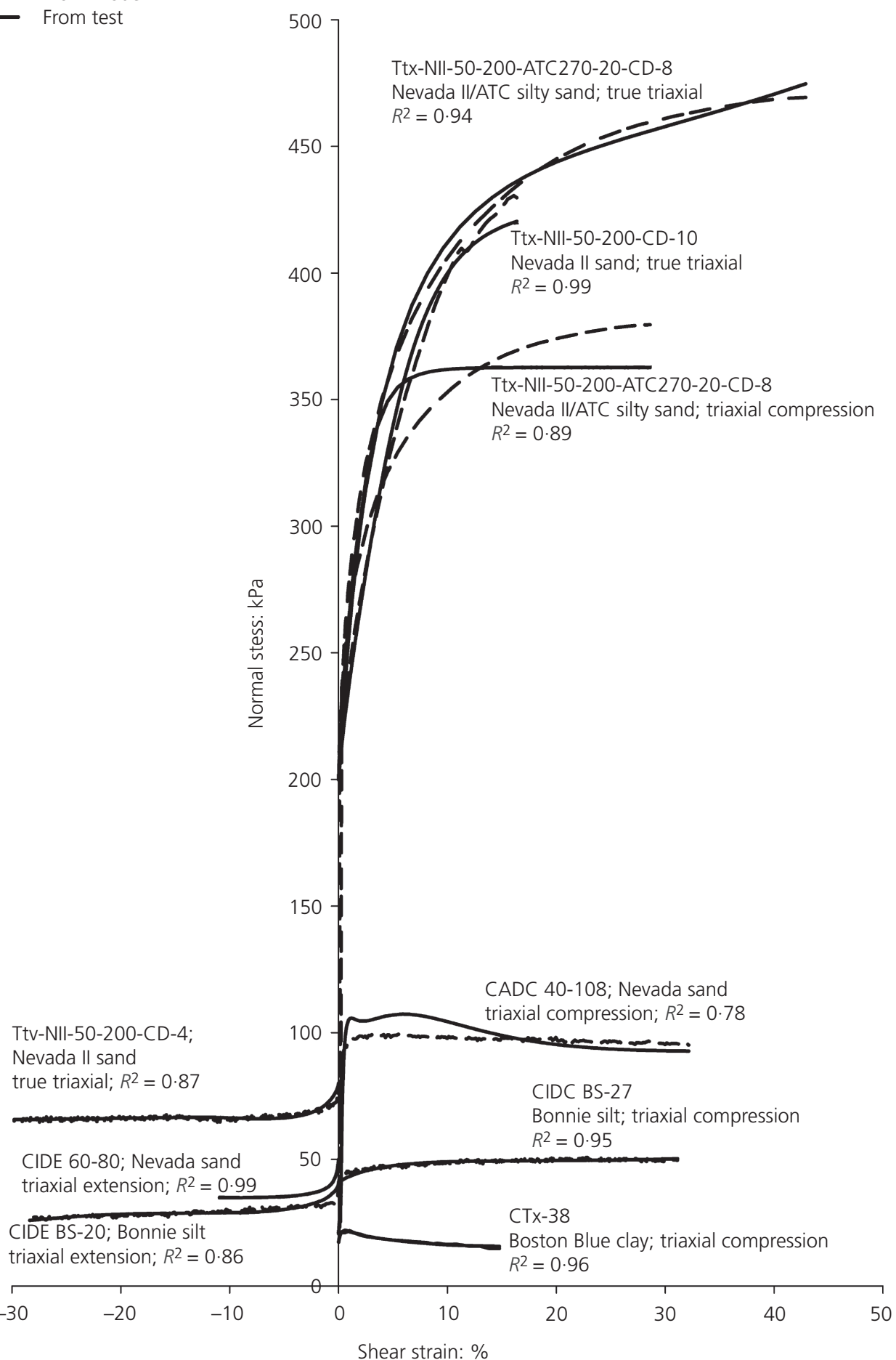

Figure 3. Measured plotted against calculated normal stress plotted against strain curves for nine sample shear tests (adapted from Joseph, 2012) 


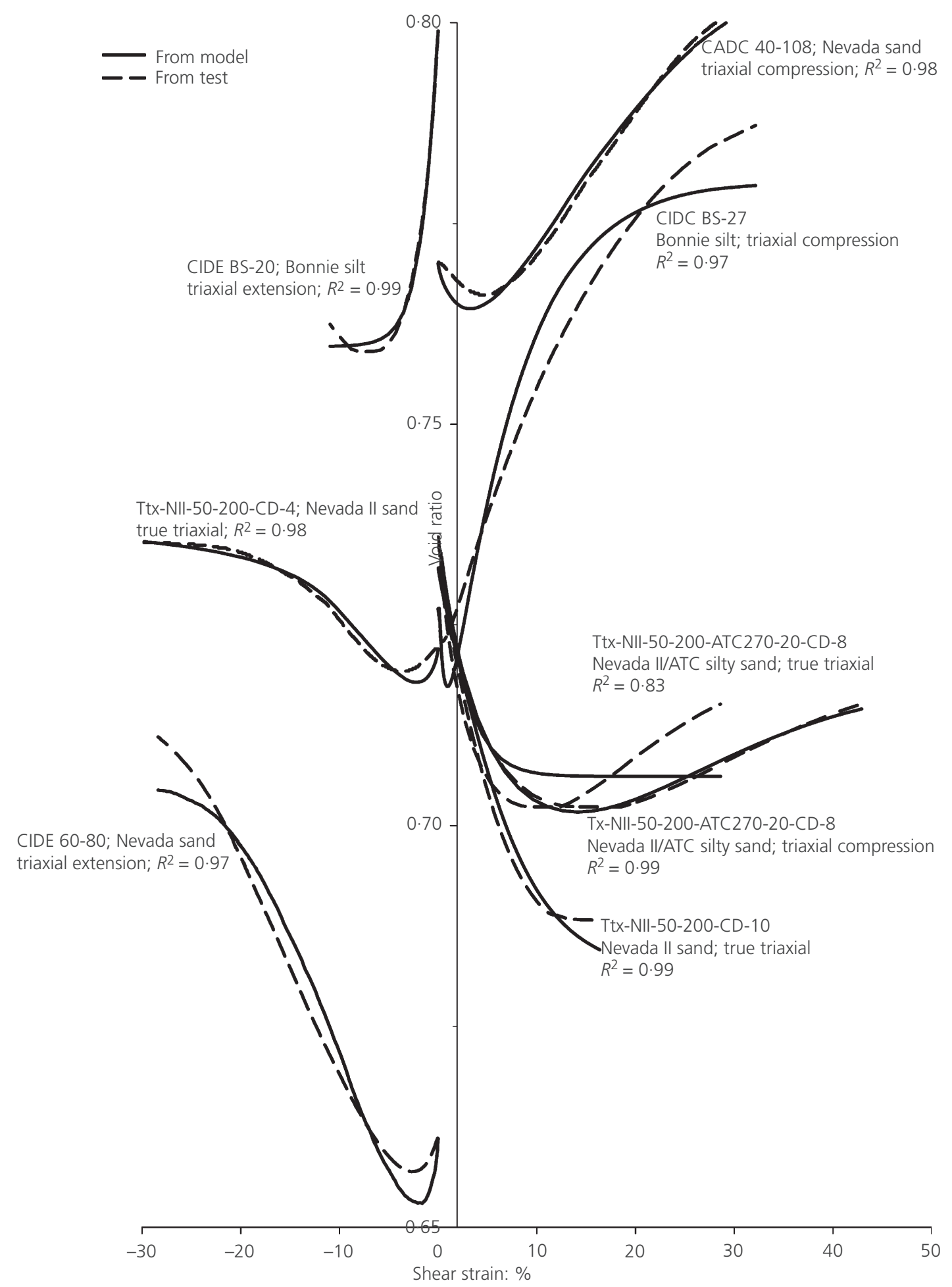

Figure 4. Measured plotted against calculated void ratio plotted against strain curves for eight sample shear tests (adapted from Joseph, 2012) 
the load is in line with the suggestion by Joseph (2013a) that the particles that move to the steady state in the Poisson process are from this minority of load-bearing particles.

The advantage of the soil deformation dynamical systems model is that it essentially describes work-hardening plasticity without the need to make any additional assumptions (which, by definition, must be specific and hence potentially erroneous) required to define an explicit flow rule or yield surface. As with discrete-element approaches, the latter are implicit in the dynamical systems model and emerge out of Equations 1a-1c. For example, for the Boston blue clay tested by Sheahan (1991), they are implicit in Figure 1.

\section{Generalisation of the model}

This section generalises the dynamical systems soil shear model to describe deformation along any stress path. The generalisation states that, for any stress path, particles move at random shear strains into the final structure corresponding to the applied stresses. In short, a friction-based dynamical system describes all soil deformation. To validate this generalisation, this paper specialises the model to the particular case of one-dimensional consolidation, which, in general, is shear along a $K$ line and, for the particular case of one-dimensional normal consolidation (NC), shear along the $K_{0}$ line. This specialisation of the generalised model results in its predicting the well-established linear relationship between void-ratio $e$ and the logarithm of the vertical effective stress $\bar{\sigma}_{\mathrm{v}}$ for normal consolidation, thereby validating the generalised model.

A close reading of the literature shows some empirical evidence for a power-law relationship between $e$ and $\bar{\sigma}_{\mathrm{v}}$. However, it is not clear that the difference from the linear relationship between $e$ and $\log \bar{\sigma}_{\mathrm{v}}$ observed in the test data from consolidation tests taken to high vertical effective stresses is not due to grain crushing (see, among others, Butterfield (1979), Cargill (1984) and Townsend (1987)).

This paper proposes that, as was the case for shear to failure, so also for any stress path - soil microstructure changes in a Poisson process; that is, particles move at random shear strains into the final structure corresponding to the final stresses for that stress path. In other words, movement of the soil particles into the final structure for any stress path occurs as a random event independent of the strain for which the particle was not in this structure. This means that the equations from Joseph (2012) for the friction-based dynamical system apply directly, even for one-dimensional consolidation.

While Joseph (2012) was explicitly for the particular case of the final structure being the steady-state flow structure, the generalisation of the model extends this behaviour to any final structure, including that resulting from one-dimensional consolidation. The parameters of the dynamical system equations take on values appropriate to the stress path acting during soil deformation. As with shear to the steady-state condition, so too for the case of one-dimensional consolidation: load-bearing particles that have not yet moved into the applicable final structure determine behaviour and parameter values. In the next section, the model is specialised for this case.

\section{Specialisation for one-dimensional consolidation}

For one-dimensional consolidation, the following additional relationships hold for the principal stresses: $\bar{\sigma}_{1}=\bar{\sigma}_{\mathrm{v}}$, where $\bar{\sigma}_{\mathrm{v}}$ is the vertical effective stress, and $\bar{\sigma}_{2}=\bar{\sigma}_{3}=\bar{\sigma}_{\mathrm{h}}=K \bar{\sigma}_{\mathrm{v}}$, where $\bar{\sigma}_{\mathrm{h}}$ is the horizontal effective stress and $K$ is the ratio of the verticalto-horizontal stress for one-dimensional conditions. For onedimensional normal consolidation, $K=K_{0}$, the coefficient of lateral earth pressure at rest.

Replacing $\bar{\sigma}_{1}$ with $\bar{\sigma}_{\mathrm{v}}$ and $\bar{\sigma}_{2}, \bar{\sigma}_{3}$ with $K \bar{\sigma}_{\mathrm{v}}$, rewrite $q$ and $\bar{p}$ defined above as

$$
q=\frac{\sqrt{2}(1-K) \bar{\sigma}_{\mathrm{v}}}{3} \text { and } \bar{p}=\frac{1+2 K}{3} \bar{\sigma}_{\mathrm{v}}
$$

Similarly for the case of shear strain $\gamma$ for the case of one-dimensional compression, $\gamma=(\sqrt{2} / 3) \varepsilon_{\mathrm{v}}$ and, consequently, $\mathrm{d} \gamma=(\sqrt{2} / 3) \mathrm{d} \varepsilon_{\mathrm{v}}$, and $\mathrm{d} q / \mathrm{d} \gamma, \mathrm{d} \bar{p} / \mathrm{d} \gamma$ become

$$
\frac{\mathrm{d} q}{\mathrm{~d} \gamma}=(1-K) \frac{\mathrm{d} \bar{\sigma}_{\mathrm{v}}}{\mathrm{d} \varepsilon_{\mathrm{v}}} \text { and } \frac{\mathrm{d} \bar{p}}{\mathrm{~d} \gamma}=\frac{(1+2 K)}{\sqrt{2}} \frac{\mathrm{d} \bar{\sigma}_{\mathrm{v}}}{\mathrm{d} \varepsilon_{\mathrm{v}}}
$$

Substitute these into Equations $1 \mathrm{a}-1 \mathrm{c}$ to get two expressions for $\mathrm{d} \bar{\sigma}_{\mathrm{v}} / \mathrm{d} \varepsilon_{\mathrm{v}}$.

From Equation 1a

$$
\begin{aligned}
\frac{\mathrm{d} \bar{\sigma}_{\mathrm{v}}}{\mathrm{d} \varepsilon_{\mathrm{v}}} & =\frac{J_{q}}{3(1-K)}\left[(1+2 K) \tan \alpha_{q} e^{-\lambda_{p} \varepsilon_{\mathrm{v}}}\right. \\
& \left.-\sqrt{2}(1-K) e^{-\lambda_{q} \varepsilon_{v}}\right] \bar{\sigma}_{\mathrm{v}}
\end{aligned}
$$

and from Equation 1b

$$
\begin{aligned}
\frac{\mathrm{d} \bar{\sigma}_{\mathrm{v}}}{\mathrm{d} \varepsilon_{\mathrm{v}}} & =\frac{\sqrt{2} J_{p}}{3(1+2 K)}\left[(1+2 K) \tan \alpha_{p} e^{-\lambda_{p} \varepsilon_{\mathrm{v}}}\right. \\
& \left.-\sqrt{2}(1-K) e^{-\lambda_{q} \varepsilon_{\mathrm{v}}}\right] \bar{\sigma}_{\mathrm{v}}
\end{aligned}
$$

Equate these two expressions and simplify to write $e^{-\lambda_{q} \varepsilon_{v}}$ in terms of $e^{-\lambda_{p} \varepsilon_{\mathrm{v}}}$ as

3. $e^{-\lambda_{q} \varepsilon_{\mathrm{v}}}=$ Const $1_{\mathrm{ocr}} e^{-\lambda_{p} \varepsilon_{\mathrm{v}}}$ 
where

$$
\text { Const }_{\text {ocr }}=\left[\frac{\frac{J_{q} \tan \alpha_{q}(1+2 K)}{(1-K)}-\sqrt{2} J_{p} \tan \alpha_{p}}{\sqrt{2}\left(J_{q}-\frac{\sqrt{2} J_{p}(1-K)}{(1+2 K)}\right)}\right]
$$

is a coefficient that is a constant for a given OCR (the terms $J_{q}, J_{p}$, $\alpha_{q}, \alpha_{p}, K$ are properties that depend only on OCR). For the case of normal consolidation, $K=K_{0}$. Correspondingly, let Const $1_{\text {ocr }}$ now simply be called Const1.

Substitute Equation 3 into Equation 1c and simplify, to get for the case of one-dimensional normal consolidation

4. $-\frac{\mathrm{d} e}{\mathrm{~d} \varepsilon_{\mathrm{v}}}=$ Const $2 e^{-\lambda_{p} \varepsilon_{\mathrm{v}}} \bar{\sigma}_{\mathrm{v}}$

where Const $2=\sqrt{2} J_{e} / 9\left[\left(1+2 K_{0}\right) \tan \alpha_{e}-\sqrt{2}\left(1-K_{0}\right)\right.$ Const1 $]$.

For one-dimensional normal consolidation, for an initial void-ratio $e_{0}$ at the start of the test, $\varepsilon_{\mathrm{v}}=\left(e_{0}-e\right) /\left(1+e_{0}\right)$, that is, $\mathrm{d} \varepsilon_{\mathrm{v}}\left[-\mathrm{d} e /\left(1+e_{0}\right)\right]$, that is, $-\left(\mathrm{d} e / \mathrm{d} \varepsilon_{\mathrm{v}}\right)=1+e_{0}$, that is, Equation 4 becomes

5. $1+e_{0}=$ Const $2 e^{-\lambda_{p}\left(\frac{e_{0}-e}{1+e_{0}}\right)} \bar{\sigma}_{\mathrm{V}}$

Solving Equation 5 for $e$

6. $e=$ Const $3-C_{c} \ln \sigma_{\mathrm{v}}$

where Const $3=e_{0}-\left[\left(1+e_{0}\right) / \lambda_{p}\right] \ln \left[\right.$ Const $\left.2 /\left(1+e_{0}\right)\right]$, the void ratio corresponding to zero vertical stress, and $C_{c}=\left(1+e_{0}\right) / \lambda_{p}$ is a constant, the compression index along the normal consolidation line.

In short, as for the case of shear to the steady state, so also for one-dimensional consolidation - particles move at random shear strains into the final $K$ structure corresponding to the final applied effective vertical stress. The model predicts that the result of this stochastic behaviour is the standard linear relationship between $e$ and $\log \bar{\sigma}_{\mathrm{v}}$ empirically found to exist for normal consolidation. In other words, it is possible to derive directly from the general model the fundamental, well-known, empirically evidenced relationship between $e$ and $\log \bar{\sigma}_{\mathrm{v}}$, thereby validating the generalised model with its underlying hypothesis.

\section{Conclusion}

This paper extends earlier work on a dynamical systems-based approach to soil shear. It proposes that soil deformation is a Poisson process - that is, a stochastic, microstructural process in which particles move at random shear strains into the final structure. This happens for any stress path - not just for paths to the steady state. This Poisson process of microstructural particle movement results in a friction-based dynamical system that describes behaviour for any stress path. From this generalisation, it follows that onedimensional consolidation, which is shear along a $K$ line, must also behave as a Poisson process - that is, as a friction-based dynamical system. This, in turn, requires that the model predict a standard, well-known, empirically observed relationship between $e$ and $\bar{\sigma}_{\mathrm{v}}$ for the case of one-dimensional normal consolidation. The fact that it does so validates the generalisation.

Work presently under way seeks to apply the dynamical systems soil shear theory to soil creep and to use the completed model in a finite-element analysis to predict deformations of an instrumented, full-scale embankment. A short self-study course on dynamical systems based soil mechanics is available online (Joseph, 2013b).

\section{Acknowledgements}

The author thanks Ms. S. Paju and Ms. J. Glendon of the Acton Memorial Library (Acton, MA, USA) for acquiring copies of papers as needed.

\section{REFERENCES}

Arlitt MF and Williamson CL (1997) Internet Web servers: Workload characterization and performance implications. IEEE/ACM Transactions on Networking 5(5): 631-645.

Arulmoli K, Muraleetharan KK, Hossain MM and Fruth LS (1992) VELACS Verification of Liquefaction Analyses by Centrifuge Studies Laboratory Testing Program Soil Data Report. The Earth Technology Corporation, Irvine, CA, USA, report to the National Science Foundation.

Butterfield R (1979) A natural compression law for soils. Géotechnique 29(4): 469-480.

Cannizzaro F, Greco G, Rizzo S and Sinagra E (1978) Results of the measurements carried out in order to verify the validity of the Poisson-exponential distribution in radioactive decay events. The International Journal of Applied Radiation and Isotopes 29(11): 649-652.

Cargill KW (1984) Prediction of consolidation of very soft soil. Journal of Geotechnical Engineering 110(6): 775-795.

Castro G, Poulos S and Leathers F (1985) Re-examination of slide of lower San Fernando Dam. Journal of Geotechnical Engineering 111(9): 1093-1107.

Castro G, Seed R, Keller T and Seed H (1992) Steady-state strength analysis of lower San Fernando Dam slide. Journal of Geotechnical Engineering 118(3): 406-427.

Desai CS (2001) Mechanics of Materials and Interfaces: The Disturbed State Concept. CRC Press, Boca Raton, FL, USA.

Desai CS, Pradhan SK and Cohen D (2005) Cyclic testing and constitutive modeling of saturated sand-concrete interfaces using the disturbed state concept. International Journal of Geomechanics 5(4): 286-294. 
Díaz-Rodríguez JA, Martínez-Vasquez JJ and Santamarina JC (2007) Strain-rate effects in Mexico City soil. Journal of Geotechnical and Environmental Engineering 135(2): 300-305.

Finno JR, Harris WW and Mooney MA (1996) Strain localization and undrained steady state of sand. Journal of Geotechnical Engineering 122(6): 462-473.

Fourie $A B$ and Tshabalala $L$ (2005) Initiation of static liquefaction and the role of $K_{0}$ consolidation. Canadian Geotechnical Journal 42(3): 892-906.

Joseph PG (2009) A constitutive model of soil based on a dynamical systems approach. Journal of Geotechnical and Environmental Engineering 135(8): 1155-1158.

Joseph PG (2010) A dynamical systems based approach to soil shear. Géotechnique 60(10): 807-812.

Joseph PG (2012) Physical basis and validation of a constitutive model for soil shear derived from micro-structural changes. International Journal of Geomechanics 13(4): 365-383.

Joseph P (2013a) Shear modeled from random particle movement. In IACGE 2013: Challenges and Recent Advances in Geotechnical and Seismic Research and Practices (Hu J, Ma J, Meneses T et al. (eds)). American Society of Civil Engineers, Reston, VA, USA, pp. 146-153.

Joseph PG (2013b) Dynamical Systems Based Soil Mechanics: A Short, Self-Study Course. See http://soilmechanics.us/dssm (accessed 25/02/2014).

Joseph PG and Graham-Eagle J (2013) Strain-rate effects in shear highlighted by a dynamical system model. International Journal of Geomechanics, http://dx.doi.org/10.1061/(ASCE) GM.1943-5622.0000360.

Lade PV and Yamamuro JA (2011) Evaluation of static liquefaction potential of silty sand slopes. Canadian Geotechnical Journal 48(2): 247-264.

LaGatta DP (1970) Residual Strength of Clays and Clay-Shales by Rotation Shear Tests. PhD thesis, Harvard University, Cambridge, MA, USA.

LaGatta DP (1971) The Effect of Rate of Displacement on Measuring the Residual Strength of Clays. US Army Corps of Engineers Waterways Experiment Station, Vicksburg, MI, USA, contract report S-71-5.

Okada Y, Sassa K and Fukuoka H (2005) Undrained shear behaviour of sands subjected to large shear displacement and estimation of excess pore-pressure generation from drained ring shear tests. Canadian Geotechnical Journal 42(3): 787-803.
O'Sullivan C (2011) Particulate Discrete Element Modelling: A Geomechanics Perspective. CRC Press, London, UK.

Poulos SJ (1981) The steady state of deformation. Journal of Geotechnical Engineering 107(5): 553-562.

Poulos S, Castro G and France J (1985) Liquefaction evaluation procedure. Journal of Geotechnical Engineering 111(6): 772-792.

Riemer MF and Seed RB (1997) Factors affecting apparent position of steady-state line. Journal of Geotechnical and Geoenvironmental Engineering 123(3): 281-288.

Shapiro S (2000) The Effects of Nonplastic Fines on the ThreeDimensional Behavior of Sand. MS thesis, Clarkson University, New York, NY, USA.

Sheahan TC (1991) An Experimental Study of the Time-Dependent Undrained Shear Behavior of Resedimented Clay Using Automated Stress Path Triaxial Equipment. ScD thesis, Massachusetts Institute of Technology, Cambridge, MA, USA.

Strogatz SH (1994) Nonlinear Dynamics and Chaos. Perseus Books Publishing, New York, NY, USA.

Townsend FC (1987) In Symposium on Consolidation and Disposal of Phosphatic and Other Waste Clays. Florida Institute of Phosphate Research, Bartow, FL, USA.

Wang G and Sassa K (2002) Post-failure mobility of saturated sands in undrained load-controlled ring shear tests. Canadian Geotechnical Journal 39(4): 821-837.

Willkomm D, Machiraju S, Bolot J and Wolisz A (2009) Primary user behavior in cellular networks and implications for dynamic spectrum access. IEEE Communications Magazine 47(3): 88-95.

Yamamuro JA and Covert KM (2001) Monotonic and cyclic liquefaction of very loose sands with high silt content. Journal of Geotechnical and Geoenvironmental Engineering 127(4): 314-324.

Yamamuro JA and Lade PV (1998) Steady-state concepts and static liquefaction of silty sands. Journal of Geotechnical and Geoenvironmental Engineering 124(9): 868-877.

Yan WM and Dong J (2011) Effect of particle grading on the response of an idealized granular assemblage. International Journal of Geomechanics 11(4): 276-285.

Zhou H and Randolph MF (2007) Computational techniques and shear band development for cylindrical and spherical penetrometers in strain-softening clay. International Journal of Geomechanics 7(4): 287-295.

\section{WHAT DO YOU THINK?}

To discuss this paper, please submit up to 500 words to the editor at journals@ice.org.uk. Your contribution will be forwarded to the author(s) for a reply and, if considered appropriate by the editorial panel, will be published as a discussion in a future issue of the journal. 